\title{
Effects of a Purified Saponin Mixture from Alfalfa on Plasma Lipid Metabolism in Hyperlipidemic Mice
}

\author{
Cong-Hong Yu, ${ }^{\#, a}$ Guo Xie, $,{ }^{\#, b}, c$ Rong-Rong He, ${ }^{c}$ Yu-Jia Zhai, ${ }^{c}$ Yi-Fang Li, ${ }^{c}$ Bun Tsoi, ${ }^{c}$ \\ Hiroshi Kurihara, ${ }^{c}$ and De-Po Yang ${ }^{*}, a$
}

${ }^{a}$ School of Pharmaceutical Sciences, Sun Yat-sen University, No. 132, East Outer Ring Road, Guangzhou Higher Education Mega Center, Guangzhou 510275, China, ${ }^{b}$ Zhongshan Institute, University of Electronic Science and Technology of China, No. 1, College Road, Shiqi District, Zhongshan 528402, China and ${ }^{c}$ Institute of Traditional Chinese Medicine and Natural Products, Jinan University, No. 601, West of Huangpu street, Guangzhou 510632, China

(Received June 8, 2011; Accepted July 25, 2011)

\begin{abstract}
Alfalfa (Medicago sativa L.) is a genus distributed widely throughout the world and considered as the richest land source of nature products. In this study, we obtained a purified saponin mixture (PSM) through a simple, repeatable and controllable separation method from alfalfa. The compounds of the saponin mixture were identified as Azukisaponin V and Soyasaponin I, which was determined as 79.26 and $20.74 \%$ respectively by HPLC-evaporative light scattering detection (ELSD) method. The effects of PSM on plasma lipid metabolism were studied in hyperlipidemic mice induced by Triton WR-1339. Results showed that PSM could significantly inhibit the increase of total cholesterol (TC), triacylglycerol (TG) and low density lipoprotein cholesterol (LDL-C) levels, and the decrease of plasma high density lipoprotein cholesterol (HDL-C) level in plasma of hyperlipidemic mice induced by Triton WR-1339. Further study demonstrated that PSM not only enhanced the activities of lipoprotein lipase (LPL) and hepatic triglyceride lipase (HTGL) in liver, but also increased their mRNA levels in hyperlipidemic mice. The results above indicate that PSM from alfalfa exerts effects on plasma lipid metabolism through elevating the activity of liver lipases in hyperlipidemic mice. The obtained data would be a good contribution to the development and utilization of alfalfa in the world.
\end{abstract}

Key words — alfalfa, saponin, Triton WR-1339, lipid metabolism

\section{INTRODUCTION}

Alfalfa (Medicago sativa L.) is a genus distributed widely throughout the world and considered as the richest land source of nature products. ${ }^{1)}$ It is traditionally used to treat hyperlipidemia in Chinese folk ${ }^{2)}$ and modern pharmacological studies indicated it has hypolipidemic activities., ${ }^{3,4)}$ Many studies proved that saponins are the main active compounds in alfalfa. ${ }^{5,6)}$ However, the relatively low content of saponins in alfalfa extracts leads to the decrease of hypolipidemic activities, and results in the unavailable medicinal and clinical usages. In this study, we obtained a purified saponin

\footnotetext{
\#These authors contributed equally to this work.

*To whom correspondence should be addressed: School of Pharmaceutical Sciences, Sun Yat-sen University, No. 132, East Outer Ring Road, Guangzhou Higher Education Mega Center, Guangzhou 510275, China. Tel. \& Fax: +86-2085221559; E-mail: 1ssydp@mail.sysu.edu.cn
}

mixture (PSM) through a simple, repeatable and controllable separation method. The compounds of the saponin mixture were identified as Azukisaponin V and Soyasaponin I (AS). The contents of each saponins was determined by HPLC method. Moreover, the hypolipidemic activities of PSM on plasma lipid metabolism were investigated in hyperlipidemic mice induced by Triton WR-1339.

\section{MATEARIALS AND METHODS}

Plant Material and Preparation of Saponin Mixture —_ Alfalfa was obtained from leaves and stems of Medicago sativa L. growing in a natural field in the Hebei province, northern China, in July 2009. The plant was verified by the herbarium of South China Botanical Garden, Chinese Academy of Sciences (Guangzhou, China). The air-dried plant material was powdered and extracted exhaus- 


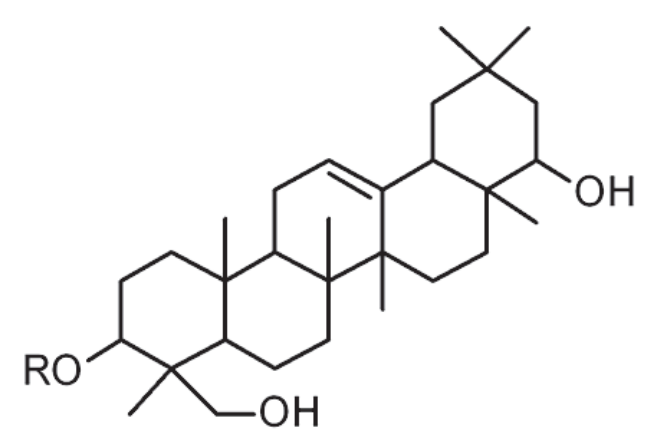

Soys aponin I R= GIcA-Gal-Rha Azukisaponin $\mathrm{V} R=\mathrm{R}$ lcA-Glc-Rha

Fig. 1. Chemical Structures of AS in Isolated Saponin Mixture from Alfalfa

PSM was obtained from alfalfa after extraction by ethanol and purification by D101 macroporous adsorption resin and ODS column chromatography. The chemical structures of PSM were identified as AS by spectrophotometer, mass spectrometer and NMR.

tively with $65 \%$ ethanol. After filtration and evaporation, the solvent was condensed under reduced pressure to obtain crude extract. The crude extract was subjected to chromatographic separation on D101 macroporous adsorption resin (Hualing Resin Co. Ltd., Shanghai, China) and eluted with water, $35 \%$, and $85 \%$ EtOH- $\mathrm{H}_{2} \mathrm{O}$ to give three fractions. The major saponins were obtained in $85 \%$ EtOH- $\mathrm{H}_{2} \mathrm{O}$ fraction. This fraction was subjected to octadecylsilane (ODS) HPLC column chromatography and eluted with 50 and $70 \%$ methanol. The $70 \%$ methanol elution was collected and solvent was removed by rotary evaporation under low temperature $\left(45^{\circ} \mathrm{C}\right)$ to yield PSM. The recovery rate of PSM was $0.99 \%$. The chemical structures of the saponin constituents were identified as AS (shown in Fig. 1) by spectrophotometer (Waters Corp., Milford, MA, U.S.A.), mass spectrometer (Bruker Daltonics, Billerica, MA, U.S.A.), and NMR (Bruker Daltonics, Billerica, MA, U.S.A.). Standard compounds of Soyasaponin I and Azukisaponin V were purchased from Tauto Biotech (Shanghai, China).

Quantification of AS by HPLC-ELSD — The quantification of AS was analyzed by HPLC-ELSD (Waters Alliance 2695-2996+SEDEX 75 ELSD), under the following conditions: column, Ultimate $^{\mathrm{TM}}$ $\mathrm{XB}^{-\mathrm{C}_{18}}(250 \mathrm{~mm} \times 4.6 \mathrm{~mm}, 5 \mu \mathrm{m})$; mobile phase, $40 \%$ acetonitrile and $60 \%$ water with $1 \%$ acetic acid; flow rate, $0.8 \mathrm{ml} / \mathrm{min}$; column temperature, $30^{\circ} \mathrm{C}$. The ELSD conditions were $100^{\circ} \mathrm{C}$ and $3.21 / \mathrm{min}$ flow rate. Quantification of each saponin was calculated by standard calibration curves. Data were obtained as average values in triplicate experi- ments.

Animals and Treatment —_ Five-week-old male Kunming mice $(18.0-20.0 \mathrm{~g})^{7,8}$ ) were purchased from Center of Laboratory Animal Science Research (Guangzhou, China). All mice were housed in plastic cages $(29.5 \mathrm{~cm} \times 17.5 \mathrm{~cm} \times 13.0 \mathrm{~cm})$ under controlled temperature $\left(23 \pm 1^{\circ} \mathrm{C}\right)$, humidity $(60 \pm 5 \%)$, with a 12 -hr day/night cycle (lights on at 06:00) and fed with a normal diet and water $a d$ libitum. The animals were allowed to acclimatize to the environment for 1 week before the experiment. Procedures for animal experiments were conducted in accordance with the Care and Use of Laboratory Animals as adopted and promulgated by the United States National Institutes of Health (NIH). Sixty mice were randomly divided into 6 groups with 10 animals each: normal control group, Triton WR-1339 model group, Fenofibrate group (FEN, $100 \mathrm{mg} / \mathrm{kg}$ ), low, middle and high doses of PSM groups $(100,200,400 \mathrm{mg} / \mathrm{kg})$. FEN was product of Laboratories Fournier S.A. (Daix, France). Mice in the PSM groups were orally administered with PSM for consecutively $7 \mathrm{~d}$. On the 7 th day, all animals except normal control group were fasted for $12 \mathrm{hr}$ prior and then treated with Triton WR-1339 (300 mg/kg), which was injected into a tail vein of mice $1 \mathrm{hr}$ after final administration. Triton WR-1339 was obtained from Sigma (St. Louis, MO, U.S.A.). Blood was drawn from heart and livers were quickly removed under anesthesia $20 \mathrm{hr}$ after Triton WR-1339 treatment. ${ }^{8)}$

Measurement of Plasma Lipids Levels Blood samples kept in a tube containing $2 \%$ sodium heparin were centrifuged at $5000 \mathrm{rpm}$ for $5 \mathrm{~min}$ to obtain plasma and stored at $-20^{\circ} \mathrm{C}$ until the assay. As previously described, ${ }^{9,10)}$ the levels of total cholesterol (TC), triacylglycerol (TG), high density lipoprotein cholesterol (HDL-C) and low density lipoprotein cholesterol (LDL-C) in plasma were enzymatically determined with commercial assay kits (Nanjing Jiancheng Bioengineering Co. Ltd., Nanjing, China).

Determination of Liver Lipases Activities

Liver samples were prepared according to the report of He et al. ${ }^{11)}$ Briefly, a 100-mg sample of tissue was removed immediately from each liver and kept in phosphate buffered saline (PBS) solution under an ice-cold condition. Then the tissues were minced with scissors and homogenized with a homogenizer at the maximum setting for $30 \mathrm{~s}$ under an ice-cold condition in $1 \mathrm{ml}$ of solution [containing $0.25 \mathrm{M}$ sucrose, $1 \mathrm{mM}$ ethylenediaminete- 
traacetic acid (EDTA), $10 \mathrm{mM}$ Trizma hydrochloric acid (Tris. $\mathrm{HCl}$ ), and $12 \mathrm{mM}$ deoxycholate, $\mathrm{pH}$ 7.4]. The homogenate was collected by centrifuging at $12000 \mathrm{rpm} 4^{\circ} \mathrm{C}$ for $20 \mathrm{~min}$ and diluted with 4 volumes of the homogenization solution, then decanted and immediately stored at $-20^{\circ} \mathrm{C}$ until assay. As previously described, ${ }^{10,11)}$ the activities of lipoprotein lipase (LPL) and hepatic triglyceride lipase (HTGL) in liver were determined using commercial assay kits (Nanjing Jiancheng Bioengineering Co. Ltd.) and expressed as units per milligram protein.

Analysis of Liver LPL and HTGL mRNAs Expression — The mRNA levels of liver LPL and HTGL were determined by quantitative realtime reverse transcription-polymerase chain reaction (RT-PCR) method using Maxima ${ }^{\mathrm{TM}}$ Synergy Brands, Inc. (SYBR) Green/Fluorescein quantitative PCR (qPCR) Master Mix (Fermentas, Hanover, $\mathrm{MD}$, U.S.A.) via $\mathrm{IQ}^{\mathrm{TM}} 5$ real-time PCR detection system (Bio-Rad, Hercules, CA, U.S.A.). The PCR primers for mice LPL mRNA were F: 5'-CTAACTGCCACTTCAACC-3' and R: 5'CAGACTTCCTGCTACGC- $3^{\prime}$, and the product size was $320 \mathrm{bp}$. The PCR primers for mice HTGL mRNA were F: 5'-TGGACGGGAAGAACAAG-3' and R: 5'-GGAGTCAATGAAGAGGTGC-3', and and the product size was $324 \mathrm{bp}$. The primers for the mice housekeeping gene 18S mRNA were F: 5'-GGGAGAGCGGGTAAGAGA-3' and R: 5'ACAGGACTAGGCGGAACA- $3^{\prime}$, and the product size was $241 \mathrm{bp}$. The final products were analyzed by $\mathrm{Ct}$ method.

Statistical Analysis — Data were presented as mean \pm S.D. The differences between experimental groups were evaluated by one-way analysis of variance (ANOVA), followed by Dunnett's test using SPSS 13.0 software. Differences between the group means were considered to be significant at $p<0.05$.

\section{RESULTS AND DISCUSSION}

In this study, PSM was obtained from alfalfa after extraction by ethanol and purification by D101 macroporous adsorption resin and ODS column chromatography. The compounds of the saponin mixture were identified as AS (shown in Fig. 1), the proportions of which were determined as 79.26 and $20.74 \%$ by HPLC-ELSD, respectively.

We further studied the effects of PSM on plasma lipid metabolism in hyperlipidemic mice induced by Triton WR-1339. As shown in Table 1, when compared with normal group, Triton WR-1339 treatment caused a marked increase in levels of plasma TC, TG, and LDL-C $(p<0.01)$ and a decrease in HDL-C level $(p<0.01)$, indicating hyperlipidemia was induced by Triton WR-1339. However, plasma TC level was significantly reduced by oral administration of PSM $(200,400 \mathrm{mg} / \mathrm{kg})$ in mice treated with Triton WR-1339. Besides, all doses of PSM remarkably decreased the levels of plasma TG and LDL-C. In contrast, PSM $(200,400 \mathrm{mg} / \mathrm{kg})$ significantly enhanced plasma HDL-C level $(p<0.05)$. The positive drug FEN $(100 \mathrm{mg} / \mathrm{kg})$ also resisted all the changes of plasma lipids caused by Triton WR-1339. Triton WR-1339 has been known to increase plasma levels of TC and TG by up-regulating 3-hydroxy-3-methylglutaryl-coenzyme A (HMG$\mathrm{CoA}$ ) reductase activity and decreasing lipases activity. ${ }^{12,13)}$ Therefore, Triton WR-1339 is widely used to study lipid metabolism and induce acute hyperlipidemia in animals to screen potential hypolipidemic drugs, in particular natural or chemical drugs. ${ }^{9,14)}$ It is well known that high levels of plasma TC, TG and LDL-C are primary risk factors for hyperlipidemia and related vascular diseases, but high plasma HDL-C level confers a protective effect against their developments. ${ }^{15)}$ Accordingly, our results demonstrated the effect of PSM on plasma

Table 1. Effect of PSM on Plasma Lipid Level and Liver Lipase Activity

\begin{tabular}{|c|c|c|c|c|c|c|}
\hline \multirow[t]{2}{*}{ Groups } & \multicolumn{4}{|c|}{$\begin{array}{c}\text { Plasma lipid level } \\
(\mathrm{g} / \mathrm{l})\end{array}$} & \multicolumn{2}{|c|}{$\begin{array}{l}\text { Liver lipase activity } \\
\text { (U/mg protein) }\end{array}$} \\
\hline & $\mathrm{TC}$ & TG & LDL-C & HDL-C & LPL & HTGL \\
\hline Normal & $113.03 \pm 9.90$ & $85.3 \pm 14.69$ & $32.19 \pm 7.53$ & $117.89 \pm 17.17$ & $1.18 \pm 0.30$ & $1.22 \pm 0.16$ \\
\hline Model & $272.53 \pm 41.03^{\# \#}$ & $570.85 \pm 85.12^{\# \#}$ & $86.06 \pm 14.21^{\# \#}$ & $89.78 \pm 21.11^{\# \#}$ & $0.85 \pm 0.23^{\#}$ & $1.05 \pm 0.17^{\# \#}$ \\
\hline FEN 100 & $138.79 \pm 10.64^{* *}$ & $58.02 \pm$ & $47.34 \pm 9.42^{* *}$ & $123.70 \pm 25.70^{* *}$ & $1.54 \pm 0.22^{* *}$ & $1.10 \pm 0.11$ \\
\hline PSM 100 & $252.73 \pm 79.87$ & $405.1 \pm 103.69^{* *}$ & $57.67 \pm 9.09^{*}$ & $95.37 \pm 11.55$ & $1.39 \pm 0.17^{* *}$ & $1.16 \pm 0.24$ \\
\hline PSM 200 & $220.71 \pm 43.90^{*}$ & $342.12 \pm 77.09^{* *}$ & $62.38 \pm 17.39^{*}$ & $122.20 \pm 33.05^{*}$ & $1.44 \pm 0.18^{* *}$ & $1.17 \pm 0.15$ \\
\hline PSM 400 & $174.21 \pm 70.85^{* *}$ & $224.79 \pm 79.95^{* *}$ & $45.13 \pm 8.52^{* *}$ & $105.86 \pm 18.49^{*}$ & $1.74 \pm 0.13^{* *}$ & $1.23 \pm 0.12^{*}$ \\
\hline
\end{tabular}

The results represent the mean \pm S.D. of values in each group $(n=10$ animals). The significance of differences from the normal control at ${ }^{\#} p<0.05,{ }^{\# \#} p<0.01$ and model control at ${ }^{*} p<0.05,{ }^{* *} p<0.01$. 


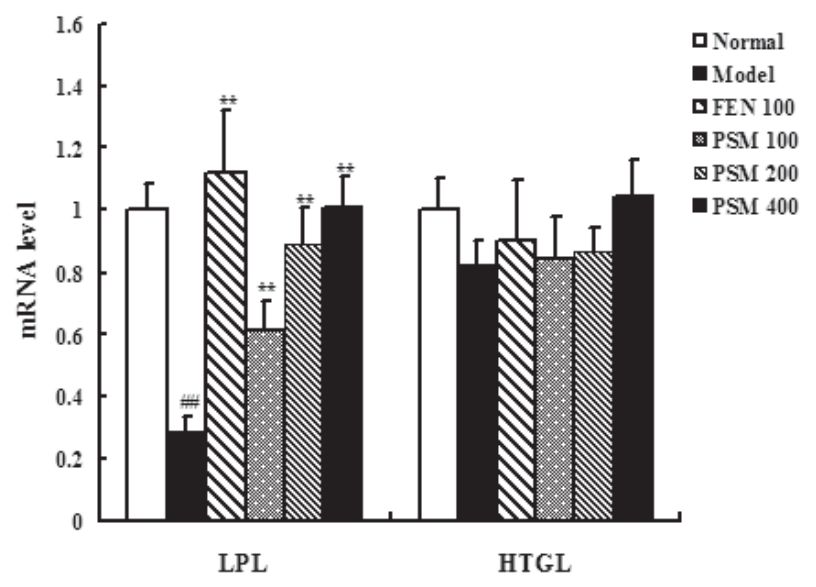

Fig. 2. Effects of PSM on mRNA Expression of Liver LPL and HTGL

The results represent the mean \pm S.D. of values in each group $(n=$ 10 animals). The significance of differences from the normal control at $\# p<0.01$ and model control at ${ }^{* *} p<0.01$.

lipid metabolism in hyperlipidemic mice.

When compared with normal mice, Triton WR1339 treatment significantly lowered the activity of liver LPL $(p<0.05)$ and HTGL $(p<0.01)$ in mice (shown in Table 1). However, FEN $(100 \mathrm{mg} / \mathrm{kg}$ ) and all doses of PSM obviously increased LPL activity $(p<0.01)$ in mice treated with Triton WR1339. Moreover, high dose of PSM $(400 \mathrm{mg} / \mathrm{kg})$ also increased HTGL activity $(p<0.01)$. Previous studies demonstrated that LPL plays a central role in lipoprotein metabolism by hydrolyzing TG. ${ }^{16)}$ Moreover, HTGL, which is another form of lipase expressed in the liver and adrenal glands, could prevent the accumulation of cholesterol and serve as a ligand that mediates the interaction of lipoproteins to cell surface receptors and proteoglycans. ${ }^{17)}$ Thus our results suggested that PSM treatment may modulate plasma lipid metabolism under hyperlipidemic condition via up-regulating liver lipases activity. Whether the enhancement of liver lipases activity was linked to their up-regulation of gene expression was continuously examined. Interestingly, our results (shown in Fig. 2) found that Triton WR1339 significantly decreased the mRNA levels of LPL $(p<0.01)$ and HTGL $(p<0.05)$ when compared to normal control, while all doses of PSM remarkably increased LPL mRNA level $(p<0.01)$ and high dose of PSM $(400 \mathrm{mg} / \mathrm{kg})$ also increased HTGL mRNA level $(p<0.05)$. The results above suggested that enhanced expression of liver lipases seems to mediate its up-regulated activity.

In conclusion, we obtained a purified saponin mixture through a simple, repeatable and control- lable separation method from alfalfa. The contents and structures of the saponin mixture were identified as $79.26 \%$ Azukisaponin V and $20.74 \%$ Soyasaponin I. The recovery rate of PSM was as high as $0.99 \%$, and it also showed significant activities on abnormal plasma lipid metabolism in hyperlipidemic mice via up-regulating the activity of liver lipases. The obtained data would be a good contribution to the development and utilization of alfalfa in the world. However, Triton WR-1339-induced hyperlipidemia in animals don't resemble the formation of human hyerlipidemia and could only used to screen potential candidate health foods or drugs. The anti-hyperlipidemia effects of PSM should be examined in further studies with more hyperlipidemia models.

Acknowledgement This work was supported by a research grant from the Cooperation Project in Industry, Education and Research of Guangdong Province and Ministry of Education of P. R. China (NO. 2010B090400398). We are very grateful to Dr. Yi Dai and Dr. Feng-Juan Tu for PSM purification and HPLC-ELSD measurement.

\section{REFERENCES}

1) Foster, S. and Duke, J. A. (2000) A field guide to medicinal plants and herbs of eastern and central North America, Houghton Mifflin Harcourt, Boston, p. 218.

2) Boon, H. and Smith, M. (2004) The complete natural medicine guide to the 50 most common medicinal herbs, Robert Rose Inc, Toronto, p. 23.

3) Francis, G., Kerem, Z., Makkar, H. P. S. and Becker, K. (2002) The biological action of saponins in animal systems: a review. Br. J. Nutr., 88, 587-605.

4) Malinow, M., McLaughlin, P., Naito, H., Lewis, L. and McNulty, W. (1978) Effect of alfalfa meal on shrinkage (regression) of atherosclerotic plaques during cholesterol feeding in monkeys 1 . Atherosclerosis, 30, 27-43.

5) Story, J., LePage, S., Petro, M., West, L., Cassidy, M., Lightfoot, F. and Vahouny, G. (1984) Interactions of alfalfa plant and sprout saponins with cholesterol in vitro and in cholesterol-fed rats. Am. J. Clin. Nutr., 39, 917-923.

6) Asgary, S., Moshtaghian, J., Hosseini, M. and Siadat, H. (2008) Effects of alfalfa on lipoproteins and fatty streak formation in hypercholesterolemic rabbits. Pak. J. Pharm. Sci., 21, 460-466.

7) Dai, X., Cui, S., Li, S., Chen, Q. and Wang, 
R. (2007) Melatonin attenuates the development of antinociceptive tolerance to delta-, but not to muopioid receptor agonist in mice. Behav. Brain Res., 182, 21-27.

8) Karl, T., Duffy, L., Scimone, A., Harvey, R. and Schofield, P. (2007) Altered motor activity, exploration and anxiety in heterozygous neuregulin 1 mutant mice: implications for understanding schizophrenia. Genes Brain Behav., 6, 677-687.

9) Kwak, Y. S., Kyung, J. S., Kim, J. S., Cho, J. Y. and Rhee, M. H. (2010) Anti-hyperlipidemic Effects of Red Ginseng Acidic Polysaccharide from Korean Red Ginseng. Biol. Pharm. Bull., 33, 468-472.

10) Zhang, J., Liu, J., Li, L. and Xia, W. (2008) Dietary chitosan improves hypercholesterolemia in rats fed high-fat diets. Nutr. Res., 28, 383-390.

11) He, R. R., Yao, N., Wang, M., Yang, X. S., Yau, C. C., Abe, K., Yao, X. S. and Kurihara, H. (2009) Effects of Histamine on Lipid Metabolic Disorder in Mice Loaded With Restraint Stress. J. Pharmacol. Sci., 111, 117-123.

12) Borensztajn, J., Rone, M. S. and Kotlar, T. J. (1976) The inhibition in vivo of lipoprotein lipase (clearing- factor lipase) activity by triton WR-1339. Biochem. J., 156, 539-543.

13) Goldfarb, S. (1978) Rapid increase in hepatic HMG CoA reductase activity and in vivo cholesterol synthesis after Triton WR 1339 injection. J. Lipid Res., 19, 489-495.

14) Khanna, A., Rizvi, F. and Chander, R. (2002) Lipid lowering activity of Phyllanthus niruri in hyperlipemic rats. J. Ethnopharmacol., 82, 19-22.

15) Braunstein, J. B., Cheng, A., Cohn, G., Aggarwal, M., Nass, C. M. and Blumenthal, R. S. (2001) Lipid Disorders: Justification of Methods and Goals of Treatment. Chest, 120, 979-986.

16) Millar, J. S., Cromley, D. A., McCoy, M. G., Rader, D. J. and Billheimer, J. T. (2005) Determining hepatic triglyceride production in mice: comparison of poloxamer 407 with Triton WR-1339. J. Lipid Res., 46, 2023-2027.

17) Santamarina-Fojo, S., Haudenschild, C. and Amar, M. (1998) The role of hepatic lipase in lipoprotein metabolism and atherosclerosis. Curr. Opin. Lipidol., 9, 211-214. 